\title{
Sensor Node Localization Based On Improved Genetic Algorithm
}

\author{
Chen Tao and Lu Min \\ Shandong Women's University, Shandong Jinan, 250300 \\ yx6785@163.com
}

\begin{abstract}
With the rapid development of science and theory study, the genetic algorithm based on natural evolution system theory research, has been applied in various fields, but the local search ability of genetic algorithm is not strong, although rely on strong global convergence can quickly reach the near optimal solution, but to search for the best solution to also want to take a long time, especially for offspring inherit father generation gene, reduced the degree of the diversity of population, makes the algorithm easy to fall into local convergence. For this purpose, the sensor node localization based on improved genetic algorithm is put forward, through multiple sensors of simple structure, low cost, integration of communication interface network group, via the network group environment to establish a sensor node sampling module node localization analysis, to pinpoint genetic algorithm search process. The simulation experiments show that the sensor node localization based on improved genetic algorithm can effectively improve the local search ability, and the search accuracy and shorten the search time, and in the distributed search, positioning speed, high precision and reliability is strong, effective for the research of genetic algorithm for better security.
\end{abstract}

Keywords: Genetic algorithm, Local search. The sensor, Node localization

\section{Introduction}

The research of our country is in a sustained and rapid development stage, the needs of the whole society is gradually expanding, especially the demand Genetic Algorithms (GA)[1]. Genetic algorithm is a new random search and optimization algorithms developed rapidly in this years, in the early 1800 s, China began to research on the genetic theory, first began to search by natural biological and natural environment of the theory, the search are is random, and select suitable means[2]. Until the advent of modern computers, scholars began by calculating and implementation of the genetic theory, and research the genetic algorithms, many scholars have noted that these natural biological showed significant resilience [3] in the process of natural evolution, genetic organisms in biological evolution has always been excellent genetic gene as the target, which making more unity in biological evolution, which become more resilient and better genes biological in nature [4]. Genetic algorithms cannot be limited by the constraints which are given in the search space, it will not require the additional conditions, such as requiring a number of associated functions satisfying derivable, differentiable or what other conditions[5]. Currently, the research on the genetic algorithm has entered into the boom stage; genetic computing has become an important branch of artificial intelligence research and direction, so that the genetic algorithm gets the more and more attention.

For the research on the stage of genetic algorithms currently, global optimization search has been basic realized basically. The characterized of genetic algorithms on this stage are simple and targeted definitely, less miscalculation and for single processing. The genetic algorithms on this stage have been successfully applied in many fields range of economic, transportation, industrial design, etc., such as DNA identification search, industrial plants, machine scheduling and the like[6].The genetic algorithms on this stage 
can control the mutation probability of selection by simulating annealing method [7], but the local search ability of the algorithm is weak, thus increasing the search time and causing genetic algorithm efficiency reducing [8]. And the algorithms on this stage are non- distributed genetic algorithms, resulting the reducing of the diversity of the population, poor scalability and other defects.

This paper presents the sensor node localization base on improved genetic algorithm, adjusting the weights of the sensor search network base on optimization generated by genetic algorithms, and do the re-initial calculations. Then do local search, create many sensors and establish an integrated network group, establish sensor node acquisition module by the network group environment, the node is analyzing located and accurately search through the sensor. Simulation results show that the sensor node localization based on improved genetic algorithm, in the integrated network environment capture sensing node accurately positioning method research under complex network group, improving the local search capability, which reduces the search time, the non-distributed becomes distributed structure, increasing the scalability, search accuracy higher and positioning speed is faster with more reliability. Which provide protection for genetic algorithms effectively [9-10].

\section{Study of Genetic Algorithm at the Present Stage}

\subsection{Genetic Algorithm at the Present Stage and the Principle of Evolution}

The characteristics of genetic algorithms at the present stage are the form of the problem to solve is not included at this stage of the algorithm. And overall optimization, gene matching is an important key of optimization [11]. Therefore, the calculation method of genetic match is from bottom to top, which is similar to the process of biological evolution, the genetic algorithm deal with the code of variable set rather than variables itself [12-13]. The theory of biological evolution framework is shown in Figure 1.

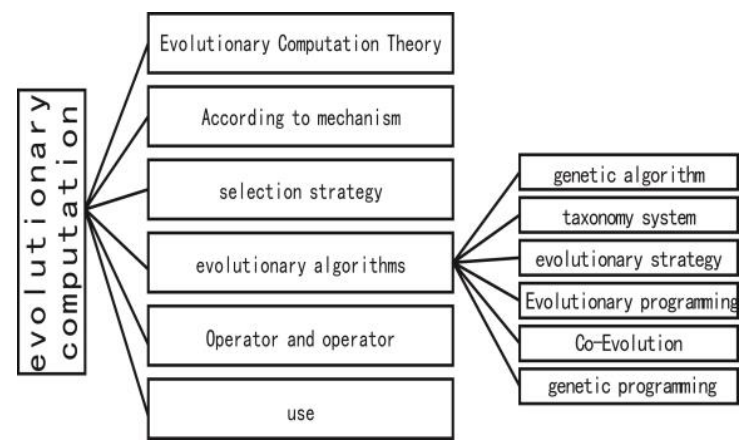

Figure 1. The Theory of Biological Evolution Frame Structure

Evolutionary theory is a subject start of the theory. Simulation based on genetic principles is the process from theory to calculating, in accordance with the law of natural selection, survival of the fittest, research and solving the algorithms and procedures which cannot be solved by traditional optimization methods of calculation in the field of science and technology, also is known as evolutionary algorithms [14]. And the scientific relationship of evolutionary computation and genetic algorithms is shown in Figure 2. 


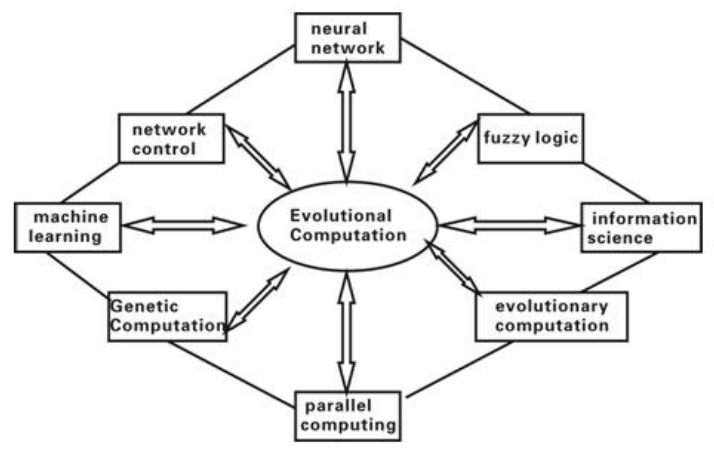

\section{Figure 2.Evolutionary Computation and Related Genetic Algorithm of Science}

In the figure above shows that the operation of strong neural network of genetic algorithm, evolution strategy emphasizes network on the control of behaviors, and genetic programming emphasizes behavioral change on the level overstep population.

\subsection{The Applications and the Step of Genetic Algorithm at the Present Stage}

At the present stage, with relatively mature of the genetic algorithms systems on genetic computing, neural networks and evolutionary computing, to imitate the process need to follow the laws of nature to judge, which need the way of selecting operators to lead scientific calculation at the present stage, but also are the different stages of the genetic computation theory and technological development. These theoretical and computation are different from the surface, but in fact they are closely related, complementary and reinforcing [15-16]. The main corresponding areas of the genetic algorithm are shown in Figure 3.

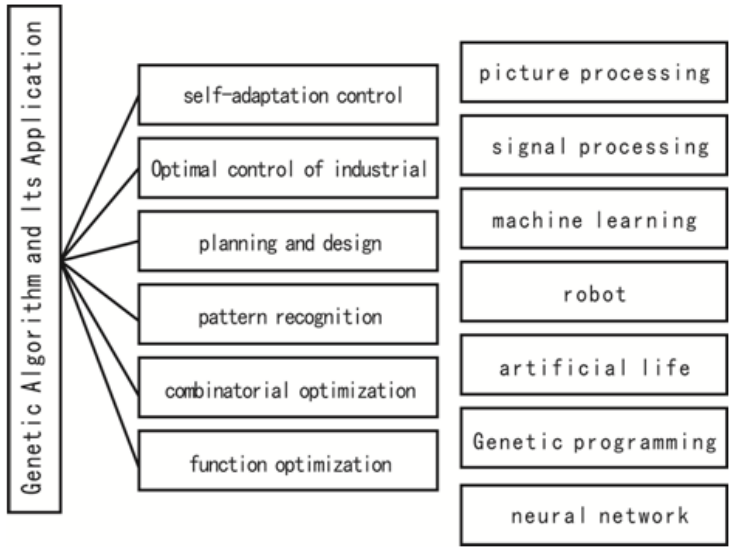

Figure 3. The Current Corresponding Areas of Genetic Algorithm

In this stage of genetic algorithms, neural networks are the high level structure to reflect the thought of brain, the evolution system have many similarities to the evolution process of the biological populations, fuzzy system is imitating the features such as the low-level brain structures. These studies theories can play an important role in each specific area, but there are some inherent limitations.

The main steps of genetic algorithm at the present stage are as follows:

1. Generating one or several initial population randomly, the initial population is composed by the characteristic strings with the fixed length.

2. Calculating the fitness value of each individual within the scope of the study. 
3. Calculating the next generation by replication, mutation, and hybridization.

4. The individual string with best performance in any generation is designated as the results of the genetic algorithm[17].

\section{Sensor Node Localization Based On the Improved Genetic Algorithm}

\subsection{The Model and Calculation of the Improved Genetic Algorithm}

Genetic algorithm proposed main draws some of the characteristics of biological evolution, simulating natural selection and genetic system of biological systems and forming an adaptive probabilistic search algorithm for global optimization. According to Darwin's "survival of the fittest" theory, in nature, only the organisms with strong ability can survive, and pass the good characteristics to their offspring by the genetic ability. And the new proposed method gives the general mathematical model[18].

Assuming the number of genetic $\mathrm{w}=1 \ldots \mathrm{W}$; $\mathrm{s}$ chromosome length is assumed to represent a symbol string $U=u 1, u 2 \ldots u n$. The combinations of allele constitute a basic understanding space formula is:

$$
K=u_{1} \times u_{2} \times \ldots \times u_{s}=\prod_{r=1}^{s} u_{r}
$$

Among them, $u_{r}(r=1,2, \ldots n)$ on behalf of a genetic, where all values are allele. Its population acclimatization formula is:

$$
K(w+1)=q_{w}(K(w), P(w))
$$

Among them, the evolution number groups of $\mathrm{w}$ is $\mathrm{K}(\mathrm{w})$, environmental labels corresponds to the number of genetic is $\mathrm{E}(\mathrm{w})$, and assume that each genetic number corresponding environment $\mathrm{E}(\mathrm{w})$ is independent, environmental ability of groups label as $\mathrm{P}(\mathrm{w})$, on the role of natural selection and genetic mechanisms $Q_{w}$ generate a new solution set groups $K(w+1)$. Therefore, the above model is revised as follows:

$$
K(w+1)=q_{w}\left(K(w), P(w), Z_{e}(w)\right)
$$

Among them, the formula of $Z_{e}(w+1)$ reflect the treatment of natural selection and genetic mechanisms in the group of natural evolution:

$$
Z_{e}(w+1)=q_{w}\left(Z_{e}(w), P(w)\right)
$$

The generation of new solution set groups can be interpreted as a stochastic process, generating a new solution set groups probability under natural selection and genetic mechanism action is:

$$
H(t)=\left\{h_{r, b}(w) \mid \sum_{b=1}^{s} h_{r, b}(w)=1\right\}
$$

Among them, ${ }^{q_{w}}$ is genetic mechanism.

\subsection{The Establishment of the Improved Sensor Network System}

The sensor module technology is a new sensor positioning technology, includes communication technology, chip manufacturing technology, batteries and energy-saving technology, sensor architecture structure extracted from the sensor structure, node hardware structure and node network protocols. The sensor network architecture shown in Figure 4. 


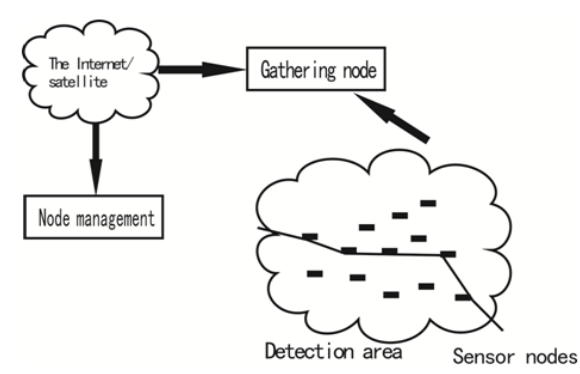

Figure 4. Sensor Network Architecture

\subsection{The Establishment of Sensor Nodes Positioning Module}

Sensor nodes are usually set deployment in the actual application environment; sensor node localization search of improved genetic algorithm is significant, then the sensor node localization architecture shown in Figure 5.

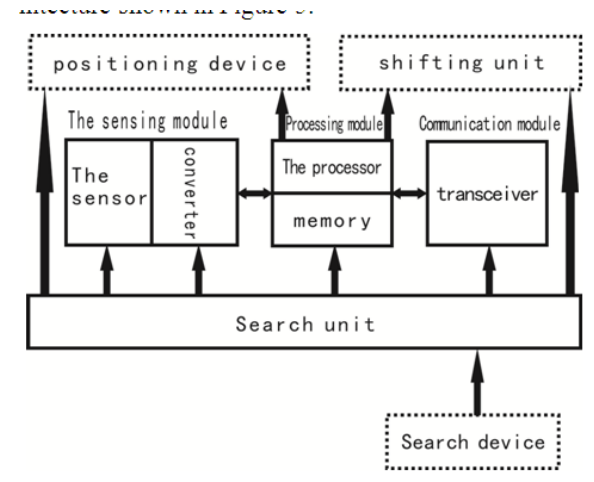

Figure 5. The System Structure of Sensor Nodes

Establishment of sensor node localization system is divided into several different modules, sensor module, processing module, communication module; and sensors, converters, processors, memory, transceiver and other processors can complete the search positioning.

Sensor nodes, node localization algorithm is the actual distance information between the search nodes or the azimuth angle information between nodes, and getting the node position location information search method. The formula of node signal search path is:

$$
H_{m}(q)=\frac{H_{w} D_{w} D_{m} \lambda^{2}}{(4 \pi)^{2} q^{2} C}
$$

Among them, $H_{w}$ is search energy, $D_{w}$ and $D_{m}$ represent the gain of the transmitting and receiving nodes, $q$ represent the system loss, $\lambda$ represents the wavelength of the search signal.

\section{Simulation and Experiments}

Injecting the technology based on improved genetic algorithm into sensor node localization system, construct the next generation of sensor nodes location search take some living creatures in the same environment as the example, set plurality of signal sensors. Do the simulation experiments base on improved genetic algorithm.

At the same time, comparing the node search ability of the genetic algorithm, the comparison of node location search capabilities among new ways and the traditional methods are shown in Figure 6. 


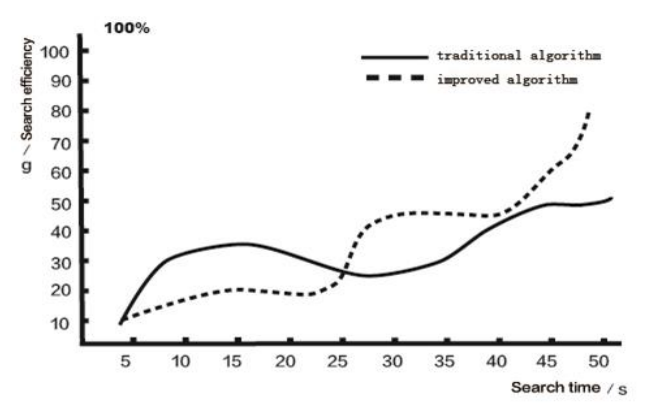

Figure 6. A New Method Is Compared With the Traditional Method of Node Location Search Ability

From the above chart shows, at the same time, the new genetic algorithm is stronger than the old method in node localization search capabilities, and relatively stable.

In the same range, the comparison of node location search in genetic algorithms, the comparison of scalable performance among the new method and the traditional method are shown in Figure 7.

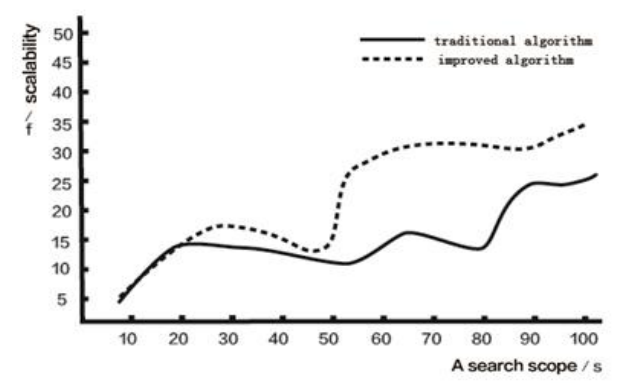

\section{Figure 7. New Methods and Extensible Performance Comparison of Traditional Method}

From the above chart shows that in the same range, the scalable ability of new genetic algorithms is larger than the old method and the search range is broader.

In the same range, the comparison of sensor nodes positioning of the genetic algorithms, the accuracy comparison among the new method and the traditional method are shown in Figure 8.

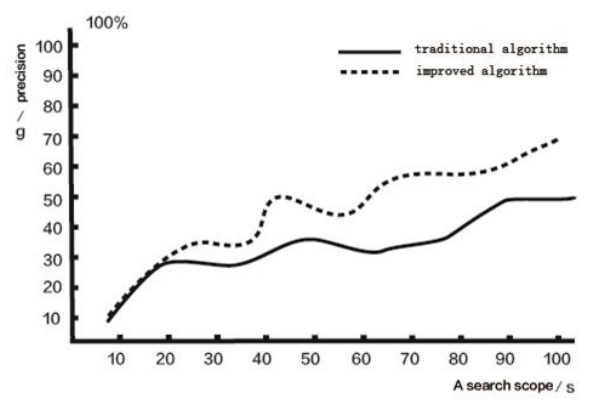

Figure 8. New Method for Node Location Search Precision Compared With Traditional Method

From the above chart shows, in the same range, the new genetic algorithm of the node searching localization accuracy is higher than the old method. 


\section{Conclusions}

Researching the sensor node localization of genetic algorithms and in the node search using genetic algorithms, there are some shortfalls in the traditional methods of genetic algorithm. The improved genetic algorithm based on positioning sensor nodes is proposed. By analysis the sensor nodes localization in the same range of biological evolution rules, choose the better genetic factor, build sensor network systems, do the nodes positioning and search process by multiple sensor modules. The simulation results that the improved genetic algorithm in the sensor nodes positioning accurate of method have solved the existing local search weak capability, long search time and with scalable aberration. Make up the shortcomings of genetic algorithm which demanding a long time to search and unstable in the relatively complex environments, which can search sensor nodes accurately with the high-speed, improving the scalability of genetic algorithms effectively. Which has provided a guarantee for the study of science.

\section{Reference}

[1] QLiangdong and H. Dengxu, “AFSA of mixed mutation operators”, Computer Engineering and Applications, vol. 44, no. 35, (2008), pp. 50-52

[2] Z. Cong and Z. Jinhua,"An improved TSP inspired crossover Operator”, Computer Engineering and Applications vol. 44, no. 9, (2008), pp. 37-39.

[3] L. Shaoxin, "Gray coding genetic algorithm inversion operation of the measurement of dynamic light scattering particle size distribution”, Computational Physics, vol. 25, no. 3, (2008), pp. 323- 330.

[4] W. -J. Gu, R. -C. Zhang and H. -C.Zhao,"On fuzzy sliding mode guidance based on self-adaptivegenetic annealing algorithm.IEEE Trans on SMC", vol. 3, no. 11, (2008), pp. 2576-2581.

[5] R. Kun and Z.Xiaoqing, "Research Based on the Improved Dynamic Programming Power Integrated System Unit", Technique s and Applications of Automation, vil. 29, no. 5, (2010), pp. 6 -8.

[6] A. Manjeshwar and D. P.Agrawal,"APTEEN, A hybrid protocol for efficient routing and comprehensive information retrieval in wireless sensor networks", Proceedings of the 3rd ACM international symposium on Mobile ad hoc networking \& computing, ACM, (2002),pp. 24-35.

[7] C.Shanshan, Z. Jianhu and L. Wenxia,“Applications of Complex genetic hybrid intelligent algorithm used in distribution network downtime optimization”, Modern Power, vol. 27, no. 1, (2010), pp. 22-25.

[8] Y. Xiangyang, "Review of wireless sensor networks", European Journal of Scientific Research, vol. 32, no. 4, (2009), pp. 444-454.

[9] X. Xu, L. Huang and X. Li,“A Fine-grained Localization Algorithm in Wireless Sensor Networks”, IEEE Wireless Communications and Networking Conference, WCNC'09, (2009).

[10] J. C. Cano and P.Manzoni,"A performance comparison of energy consumption for mobile ad hoc network routing protocols", Proceedings of the 2nd ACM international conference on Wireless sensor networks and applications. ACM, (2003), pp. 122-131.

[11] E. Coppola Jr., F. Szidaroszky, M.Poulton, and E. Charles, "Artificial Neural Network Approach for Predicting Transient Water Levels in a Multilayered Groundwater System under Variable State , Pumping and Climate Conditions", J. of Hydrologic Engineering vol. 8, no.6, (2003), pp. 348-360.

[12] C. L. Barrett, S. J. Eidenbenz, L. Kroc, et al.,"Parametric probabilistic sensor network routing, Modeling, Analysis and Simulation of Computer and Telecommunication Systems", 2000. Proceedings. 8th International Symposium on. IEEE, (2000), pp. 57-64.

[13] S. K. Das, B. S.Manoj,C. S. R. Murthy,“A dynamic core based multicast routing protocol for ad hoc wireless networks, Parallel and Distributed Processing Symposium”, International. IEEE Computer Society, vol. 2, (2002), pp. 0195b-0195b.

[14] N. Minar, K. H. Kramer and P.Maes, "Cooperating mobile agents for dynamic network routing, Software agents for future communication systems", Springer Berlin Heidelberg, (1999), pp. 287-304.

[15] Z. Zhang, X. Shao and W. Ding, "MPLS ATCC, an Active Traffic and Congestion Control Mechanism in MPLS。2001 International Conferences on Info-tech and Info-net (ICII2001) ”, vol. 5, (2001), pp. 205-210.

[16] M. Karamouz, R.Kerchian and B. Zahrie," Monthly Water Resources and Irrigation Planning”, Case Study of Conjunctive Use of Surface and Groundwater Resources", J. of Irrigation and Drainage Engineering, vol. 130, no. 5,(2004), pp. 391-402.

[17] R. C. Eberhart and Y.Shi,"Particle swarm optimization: developments, applications and resources, Evolutionary Computation, 2001", Proceedings of the 2001 Congress on. IEEE, vol. 1, (2001), pp. 81-86. 
[18] M. Saad, M. Izuan, A.Zulkarnain, "Performance analysis of random-based mobility models in MANET routing protocol", Microcontroller and Embedded Systems Applications,no. 8, (2008), pp. 8-12

\section{Authors}

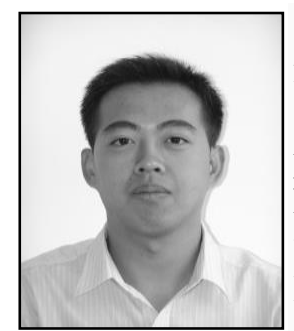

Chen Tao, received the master's degree in computer engineering from Computer Science School of Shandong University, China in 2009. He is now severing in Shandong Women's University, with the professional title of experimentalist. Besides daily teaching activities, he is currently researching on computer network and database, as well as responsible for several scientific study projects in department level.

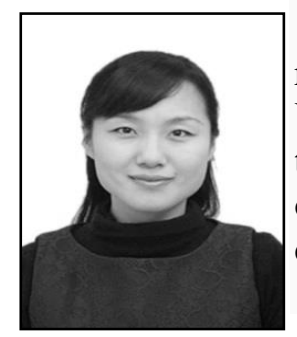

Lu Min, graduated from Shandong Formal University, and received the master degree in 2014. She is now working at Shandong Women's University, with the title of lecturer. She is engaged in teaching and informational student management, and has presided over and participated in several research tasks of provincial and departmental level research project. 